\title{
IMPORTANCIA DE LAS EXPERIENCIAS PREVIAS EN LA VOCACIÓN Y ELECCIÓN DE LA TITULACIÓN DE MAESTRO CON MENCIÓN EN EDUCACIÓN FÍSICA
}

\author{
IMPORTANCE OF PREVIOUS EXPERIENCES IN THE CALL AND CHOICE OF \\ THE TEACHER DEGREE IN PHYSICAL EDUCATION
}

\section{IMPORTÂNCIA DAS EXPERIÊNCIAS ANTERIORES NA VOCAÇÃO E ELEIÇÃO DO GRAU DE PROFESSO COM MENÇÃO EM EDUCAÇÃO FÍSICA}

\author{
Sebastián Feu*, Manuel Vizuete *, Ernesto De la Cruz-Sánchez **, \\ Amalia Gragera-Alonso***
}

\section{Palabras clave:}

Educación Física.

Docentes.

Acontecimientos

que cambian la vida.

Deportes.

Elección de

profesión.
Resumen: Los objetivos del estudio fueron determinar cuál es la influencia de la práctica deportiva realizada en la etapa escolar y en la actualidad en la elección de la especialidad de profesor de Educación Física. En este estudio contó con 234 participantes con grado de profesores de ducación primaria, donde un $67,5 \%$ eran mujeres y un $32,5 \%$ eran hombres. Se ha realizado un estudio cuantitativo mediante encuesta, de tipo descriptivo e inferencial. El alumnado de la especialidad de maestro de Educación Física se caracteriza por poseer mayores recursos previos para diseñar un calentamiento, un programa de acondicionamiento físico o practicar deportes y actividades físicas en la naturaleza. Los estudiantes que se percibe que tienen más capacidades para participar y organizar actividades de danza y baile se han decantado por las otras especialidades, siendo las mujeres las que más capaces se sienten en este tipo de tarea. Las capacidades adquiridas en la escuela para practicar actividad física, el género y la experiencia personal en actividades físicas de ocio influyen en la vocación para ser de profesor de educación física.

Palavras-chave: Educação Física. Docentes.

Acontecimentos que mudam a vida. Esportes.

Escolha da profissão.

\section{Keywords:}

Physical education.

Teacher.

Life-changing

events.

Sports.

Career choice.
Resumo: Os objetivos do estudo foram determinar qual a influência da prática desportiva realizada em meio escolar e na atualidade, na escolha da especialidade de professor em Educação Física. Neste estudo participaram 234 participantes com o grau de professores de educação primária, em que 67,5\% eram mulheres e 32,5\% homens. Realizou-se um estudo quantitativo utilizando uma pesquisa do tipo descritiva e inferencial. Os alunos com a especialidade de professores de Educação Física caraterizam-se por terem mais recursos para projetarem um aquecimento, um programa em ginástica ou em desportos e atividades físicas na natureza. Os estudantes que se percebe terem mais capacidades para participar de e organizar atividades de dança e baile inclinaram-se pelas outras especialidades, sendo as mulheres as que se sentem mais capazes neste tipo de tarefa. As competências adquiridas na escola para a prática de atividade física, o género e a experiência pessoal em atividades físicas de lazer influenciam a vocação para ser professor de Educação Física.

Abstract: The aim of this study is to determine how sports practice during school years and presently affects teachers' beliefs and perceptions about Physical Education. The descriptive and survey-based inferential study included 234 Primary Education student teachers (67.5\% women and $32.5 \%$ men). Physical Education students are characterized by having higher previous resources to design warming-up routines, a physical conditioning program of sports and physical activities. Students who perceive themselves as more capable of participating and organizing dance-related activities chose other areas, and women felt more capable to conduct that task. Sport experience and beliefs acquired during school years for physical activity, gender and personal experience in spare-time physical activities impact one's call to be a Physical Education teacher.
* Universidad de Extremadura. Extremadura, Espanha. E-mail: sfeu@unex.es

** Universidad de Murcia. Murcia, Espanha.

E-mail: erneslacruz@um.es

${ }^{* * *}$ Escuela de Artes y Oficios Adelardo Covarsí. Badajoz. Espanha. E-mail: malavara18@hotmail.com

Recebido em: 11-10-2015 Aprovado em: 14-06-2016 (c) (1) (8) Licence 


\section{INTRODUCCIÓN}

La presencia de maestros especialistas en la enseñanza primaria europea es práctica habitual en materias como educación física, música, arte e idiomas (HALL, 2000). Estos profesionales comienzan su formación antes de iniciar sus estudios al estar en contacto con contenidos de su materia tanto en el ámbito escolar como extraescolar. A través del proceso de socialización ocupacional los nuevos miembros de la profesión descubren los recursos profesionales (VAN MAANEN; SCHEIN, 1979). La formación de profesionales se desarrolla a través de distintos modelos formativos: formales, no formales e informales (COLOM, 2005). En el caso de los docentes, la formación específica se desarrolla de acuerdo con la regulación del sistema educativo universitario, acreditando al profesor la formación necesaria para ejercer la docencia en el sistema educativo; mientras que la educación no formal se realiza mediante actividades formativas complementarias que contribuyen a la actualización y especialización docente a través de actividades como cursos, congresos, seminarios,...; por último, la educación informal son actividades desarrolladas por el docente fuera del sistema formativo y que ofrecen un amplio abanico de posibilidades (RODRÍGUEZ; FERNÁNDEZ, 2005). Los docentes utilizan en alto grado experiencias de aprendizaje informal (LOHMAN, 2006).

En el caso de las diferentes especialidades de la titulación de maestro, la amplia gama de experiencias que contribuyen a la formación informal abarcan desde la infancia hasta los momentos en los que se produce la formación académica; y por tanto engloban desde sus experiencias en el sistema educativo como alumnos (EIRÍN; GARCÍA-RUSO; MONTERO, 2009), a la experiencia extraescolar pasada y presente, así como la relación en todo este proceso con otros profesionales, la familia, internet,... Se trata de un proceso formativo no estructurado, basado en vivencias personales, que influye en el futuro docente (BURKE, 1987; EIRÍN, et al., 2009). En la socialización del profesor interviene su propia biografía, construida desde sus experiencias previas a la institución formativa, llegando a formar parte de sus creencias y escalas de valores (JORDELL, 1987; PORLAN; RIVERO, 1998; RANDALL; MAEDA, 2010; RYAN; BRIDGES; YERG, 2000).

Una de las menciones de la titulación de grado de maestro con mayor influencia y estímulo social y cultural es la de Educación Física. Los futuros maestros de educación física, al igual que los entrenadores deportivos escolares, asimilan su cultura docente no sólo mediante vías formales y académicas, sino que, en un elevado grado, se socializan en su profesión a través del aprendizaje vicario adquirido desde sus primeras experiencias de educación física escolar y del deporte extraescolar (FEU; IBÁÑEZ; GOZALO, 2010; FEU; IBÁÑEZ; LORENZO; JIMÉNEZ; CAÑADAS, 2012; VALTONEN; REUNAMO; HIRVENSALO; RUISMÄKI, 2015). Las experiencias previas satisfactorias, sobre todo en los deportes de equipo, favorecen que los futuros docentes adquieran una percepción de competencia motriz que les hace creer que pueden ser más competentes en la profesión de maestro de educación física, ya que estos contenidos son los que creen que predominan en la educación primaria y secundaria (VALTONEN et al., 2015). El éxito y el gusto por el deporte parece ser uno de los motivos para iniciar los estudios de profesor de educación física (O'BRYANT; O'SULLIVAN; RAUDENSKY, 2000). 
De esta forma, la necesidad de tener en cuenta las creencias e ideas previas que tienen los docentes de educación física al inicio de su formación inicial ha sido considerada por numerosos estudios (LATORRE, 2007). Los estudiantes de formación del profesorado llegan a sus estudios con una opinión idealizada de la función docente contaminada por sus experiencias previas (SHKEDI; LARON, 2004). Por ello, este estudio plantea, desde esta perspectiva, dos objetivos: el primero, determinar cómo influye la práctica deportiva en la edad escolar y en el momento actual en la elección de la especialidad de maestro de educación física; y el segundo, determinar qué capacidades relacionadas con la educación física adquirieron los futuros maestros en su etapa escolar para una práctica autónoma de la actividad física y analizar su influencia en la elección de la especialidad de maestro.

\section{MÉTODO}

Se realizó un estudio de corte cuantitativo y descriptivo de poblaciones mediante encuestas con muestras probabilísticas y transversal (MONTERO; LEÓN, 2007); además puede considerarse como un estudio comparativo al indagar en las diferencias que se producen en la elección de especialidad de maestro en función de unas características poblaciones, el género y las experiencias previas (ATO; LÓPEZ; BENAVENTE, 2013).

\subsection{Participantes}

En el estudio han participado 234 estudiantes del Grado de Maestro de $3^{\circ}$ curso de Educación Primaria, el $67.5 \%$ mujeres y el $32.5 \%$ hombres. El criterio de selección de este curso obedece a que es el curso donde tienen la primera asignatura relacionada con la educación física escolar y es el curso donde deben elegir entre cuatro itinerarios de intensificación o especialidad. Se acudió a todo el censo de la Facultad de Educación que cumplía con la organización académica descrita como criterio de inclusión, 320 estudiantes, obteniendo en este estudio un $3.3 \%$ de error para un $95 \%$ de nivel de confianza.

\subsection{Instrumento}

Se diseñó un instrumento para recoger información sobre la práctica deportiva realizada en la etapa escolar y en la actualidad así como capacidades adquiridas en la educación física que en su etapa escolar para una práctica autónoma de la actividad física. La validez de contenido se determinó través de un grupo de nueve expertos, que valoraron los ítems en una escala Likert de 1 a 10 puntos, obteniendo un promedio de acuerdo adecuado, superior a 8 en todos los ítems (BULGER; HOUSNER, 2007).

Las capacidades adquiridas, en la etapa escolar, para una práctica autónoma de la actividad física se recogieron a través de diez ítems contestados en una escala likert de 5 puntos ( $1=$ Totalmente en desacuerdo; $2=$ En desacuerdo; $3=\mathrm{Ni}$ de acuerdo ni desacuerdo; 4 = De acuerdo; 5 = Totalmente de acuerdo); estos ítems obtuvieron un fiabilidad óptima, $\alpha=.86$, (NUNNALLY; BERNSTEIN, 1994). Se hicieron preguntas cerradas para determinar las características de los participantes, la elección de la especialidad a 
realizar en cuarto curso y su participación en la actividad física, tanto en la edad escolar como en la actualidad.

\subsection{Análisis estadístico}

Se realizó un análisis descriptivo de los ítems en función de las variables género, participación en el deporte extraescolar y práctica semanal de actividad física deportiva. Se estudio la asociación entre el sexo y la intención de estudiar la especialidad de educación física u otra especialidad a través de una tabla de contingencia, analizándose esta asociación a través de los estadísticos Chi-cuadrado y Phi de Cramer, las relaciones entre casillas se estudiaron a través de los residuos tipificados corregidos $(R T C)$. Por último se realizó un análisis inferencial de los conocimientos y capacidades adquiridas en la etapa pre-universitaria a través de pruebas no paramétricas, esta decisión se tomo al no cumplirse el supuesto de normalidad y al emplear variables ordinales. Las diferencias entre grupos en el caso de variables de más de dos grupos se calcularon estableciendo las diferencias grupo a grupo junto con la aplicación de la corrección de Bonferroni para la variable años de práctica extraescolar $(p<.005)$ y para los días de práctica deportiva a la semana en la actualidad $(p<.008)$.

\section{RESULTADOS}

En el estudio han participado 71 estudiantes, el $13.3 \%$ mujeres y $65.8 \%$ hombres, que declararon su intención finalizar sus estudios de maestro especializándose en el área de educación física y 163 estudiantes con la intención de estudiar otras especialidades, la distribución por sexos guarda la proporción de estudiantes matriculados en la titulación en la universidad en la que se ha llevado a cabo el estudio, ver Tabla 1. El $70.4 \%$ de los que prefieren hacer la especialidad de educación física son hombres, mientras que el $84 \%$ de las personas que prefieren hacer otras especialidades son mujeres; los resultados indican que hay una asociación significativa entre el sexo y la especialidad ( $X^{2}=66.92$; $p<.01)$ que se confirma a través del estadístico Coeficiente de Phi $(\phi=.562)$, los residuos tipificados corregidos confirman que en la especialidad de educación física hay más hombres $(R T C=8.2)$.

La elección de especialidad es más frecuente en los estudiantes con experiencia de práctica físico deportiva en el momento de la recogida de datos $\left(X^{2}=66.92 ; p<.01 ; V c=.516\right)$, pero no parece condicionada por la cantidad práctica físico deportiva realizada en la edad escolar ( $p>$.05). Los residuos tipificados corregidos informa que hay más casos de los esperados de alumnos que eligen educación física entre los que realizan cuatro o más días de práctica semanal $(R T C=5$ y 5.2). Los estudiantes que van a elegir la especialidad de educación física realizan más actividad física semanal que los de otras especialidades $(p<.01)$, siendo los hombres los que significativamente practican más actividad física semanal $\left(X^{2}=44.08 ; p<.01\right.$; $V c=.434)$. Hay más hombres que realizan cuatro o más días de actividad física semana ( $R T C=$ 3.4 y 4.7$)$ 
Tabla 1 - Porcentaje y recuento dentro de la especialidad de maestro en función del género, la participación en la actividad física extraescolar en la edad escolar y en la actualidad

\begin{tabular}{lcccccccc}
\hline & \multicolumn{4}{c}{ Especialidades } & \multicolumn{3}{c}{ Género } \\
\cline { 3 - 9 } & \multicolumn{3}{c}{ Otras } & \multicolumn{2}{c}{ Educación Física } & Mujeres & \multicolumn{2}{c}{ Hombres } \\
\cline { 3 - 9 } & $\%(\mathrm{n})$ & RTC & $\%(\mathrm{n})$ & RTC & $\%(\mathrm{n})$ & RTC & $\%(\mathrm{n})$ & RTC \\
\hline Género & & & & & & & & \\
& Mujeres & $84.0(137)$ & 8.2 & $29.6(21)$ & -8.2 & & & \\
& Hombre & $16.0(26)$ & -8.2 & $70.4(50)$ & 8.2 & & & \\
\end{tabular}

Deporte extraescolar en la edad escolar

$\begin{array}{rcccccccc}\text { No he participado } & 9.8(16) & 2.3 & 1.4(1) & -2.3 & 8.9(14) & 1.4 & 3.9(3) & -1.4 \\ 1-2 \text { años } & 4.9(8) & 1.3 & 1.4(1) & -1.3 & 2.5(4) & -1.5 & 6.6(5) & 1.5 \\ 3-4 \text { años } & 3.7(6) & -.2 & 4.2(3) & .2 & 2.5(4) & -1.5 & 6.6(5) & 1.5 \\ 5-6 \text { años } & 9.8(16) & -.3 & 11.3(8) & .3 & 10.8(17) & .4 & 9.2(7) & -.4 \\ \text { Más de 6 años } & 71.8(117) & -1.6 & 81.7(58) & 1.6 & 75.3(119) & .3 & 73.7(56) & -.3\end{array}$

Actividad física semanal en la actualidad

\begin{tabular}{rcccccccc}
1 día & $31.3(51)$ & 3.7 & $8.5(6)$ & -3.7 & $32.3(51)$ & 4.1 & $7.9(6)$ & -4.1 \\
$2-3$ días & $48.5(79)$ & -3.9 & $21.1(15)$ & 3.9 & $44.3(70)$ & 1.9 & $31.6(24)$ & -1.9 \\
$4-5$ días & $19.6(32)$ & -5.2 & $53.5(38)$ & 5.2 & $22.8(36)$ & -3.4 & $44.7(34)$ & 3.4 \\
+5 días / semana & $0.6(1)$ & -5 & $16.9(12)$ & 5 & $0.6(1)$ & -4.7 & $15.8(12)$ & 4.7 \\
\hline \multicolumn{7}{c}{$\begin{array}{c}{ }^{*} p<05 ; * * * 0.01 \\
\text { Fuente: Elaboración propia }\end{array}$}
\end{tabular}

Se les preguntó a los estudiantes por la percepción de las capacidades adquiridas en su periodo escolar, pre-universitario, en el área de educación física. Los ítems fueron contestados en una escala likert de 5 puntos (de 1 a 5 puntos) pudiéndose comprobar que la puntuación más alta fue participar en deportes de equipo $(M=4.13 \pm 1.01)$, deportes individuales $(M=3.88$ $\pm 1.15)$ y participar en deportes recreativos al menos una vez por semana $(M=3.83 \pm 1.14)$. Las medias más bajas fueron planificar actividades de varios días en la naturaleza ( $M=2.72$ \pm 1.12 ), planificar actividades físicas en la naturaleza con seguridad ( $M=2.74 \pm 1.10)$, diseñar un programa de acondicionamiento físico $(M=2.78 \pm 1.03)$ y organización de una competición deportiva ( $M=3.16 \pm 1.09$ ). En cuanto al género, los chicos se sienten más competentes en la mayoría de ítems excepto en la organización de actividades coreográficas de danza o baile donde las chicas ( $M=3.66 \pm 1.22$ ) se sienten más capaces (Tabla 2).

Los futuros alumnos de la especialidad de educación física presentan significativamente $(p<.05 / p<.01)$ mayores puntuaciones en todas las capacidades adquiridas en su etapa preuniversitaria exceptuando la organización de actividades coreográficas y de danza donde se sienten más capaces los estudiantes con intención de estudiar otras especialidades. 
Tabla 2 - Descriptivos de las capacidades adquiridas para la práctica de actividad física tras la etapa pre-universitaria y análisis de diferencias entre grupos

\begin{tabular}{|c|c|c|c|c|c|c|c|}
\hline & \multirow[t]{2}{*}{ Total } & \multicolumn{3}{|c|}{ Género } & \multicolumn{3}{|c|}{ Especialidad } \\
\hline & & Mujer & Hombre & & Otras & $\begin{array}{l}\text { Educación } \\
\text { Física }\end{array}$ & \\
\hline Soy capaz de... & $M \pm$ D.t. & $M \pm$ D.t. & $M \pm$ D.t. & $p$ & $M \pm$ D.t. & $M \pm$ D.t. & $p$ \\
\hline Diseñar un calentamiento & $3.28 \pm 1.00$ & $3.11 \pm .10$ & $362 \pm .92$ & $* \star$ & $3.04 \pm .94$ & $3.83 \pm .93$ & ** \\
\hline $\begin{array}{l}\text { Diseñar mi programa de } \\
\text { Acondicionamiento Físico }\end{array}$ & $2.78 \pm 1.03$ & $2.54 \pm .98$ & $3.28 \pm .95$ & $* \star$ & $2.56 \pm .96$ & $3.30 \pm .99$ & ** \\
\hline $\begin{array}{l}\text { Planificar actividades de varios } \\
\text { días en naturaleza }\end{array}$ & $2.72 \pm 1.12$ & $2.66 \pm 1.12$ & $2.84 \pm 1.12$ & - & $2.61 \pm 1.10$ & $2.97 \pm 1.15$ & * \\
\hline $\begin{array}{l}\text { Planificar actividades Física } \\
\text { naturaleza con seguridad }\end{array}$ & $2.74 \pm 1.10$ & $2.61 \pm 1.10$ & $3.01 \pm 1.05$ & $* \star$ & $2.60 \pm 1.06$ & $3.07 \pm 1.12$ & ** \\
\hline $\begin{array}{l}\text { Participar en actividades Físicas } \\
\text { en naturaleza }\end{array}$ & $3.42 \pm 1.20$ & $3.32 \pm 1.21$ & $3.64 \pm 1.14$ & - & $3.30 \pm 1.19$ & $3.70 \pm 1.16$ & * \\
\hline $\begin{array}{l}\text { Participar en alguna modalidad } \\
\text { de deportes de equipo }\end{array}$ & $4.13 \pm 1.01$ & $3.86 \pm 1.06$ & $4.68 \pm .61$ & $* \star$ & $3.86 \pm 1.05$ & $4.75 \pm .55$ & $\star \star *$ \\
\hline $\begin{array}{l}\text { Participar en alguna modalidad } \\
\text { deportiva individual }\end{array}$ & $3.88 \pm 1.15$ & $3.63 \pm 1.22$ & $4.41 \pm .77$ & $\star \star$ & $3.60 \pm 1.19$ & $4.54 \pm .71$ & ** \\
\hline $\begin{array}{l}\text { Organizar una competición } \\
\text { deportiva o juegos }\end{array}$ & $3.16 \pm 1.09$ & $2.91 \pm 1.08$ & $3.67 \pm .93$ & ** & $2.91 \pm 1.05$ & $3.73 \pm .97$ & ** \\
\hline $\begin{array}{l}\text { Participar en deporte recreativo } \\
\text { al menos una vez a la semana }\end{array}$ & $3.83 \pm 1.14$ & $3.65 \pm 1.22$ & $4.21 \pm .85$ & $* *$ & $3.57 \pm 1.20$ & $4.42 \pm .73$ & ** \\
\hline $\begin{array}{l}\text { Organizar y participar en } \\
\text { actividades con coreografías de } \\
\text { danza y baile }\end{array}$ & $3.34 \pm 1.24$ & $3.66 \pm 1.22$ & $2.67 \pm 1.01$ & $* *$ & $3.50 \pm 1.23$ & $2.97 \pm 1.21$ & ** \\
\hline
\end{tabular}

Por último, se analizaron las capacidades alcanzadas en función de la práctica físicodeportiva extraescolar (Tabla 3), encontrándose que los estudiantes con más de seis años de práctica en el deporte extraescolar se sienten más capaces que los que no han practicado nunca para, diseñar un calentamiento, diseñar un programa de acondicionamiento físico, practicar algún deporte individual y organizar alguna competición deportiva, $(p<.005)$. Por otro lado, los estudiantes que más días a la semana practican actividad física en la actualidad se auto-perciben más capaces en todos las capacidades indicadas en la tabla 5, ( $p<.008)$. En la organización de actividades coreográficas y de danza no hay diferencias significativas en función de la práctica de actividad física semanal. 
Tabla 3 - Capacidades alcanzadas en el área de educación física etapa pre-universitaria en función de la actividad física extraescolar

\begin{tabular}{|c|c|c|c|c|c|c|c|c|c|c|c|}
\hline \multirow{3}{*}{$\begin{array}{l}\text { Soy capaz } \\
\text { de... }\end{array}$} & \multicolumn{6}{|c|}{ Práctica deportiva extraescolar } & \multicolumn{5}{|c|}{$\begin{array}{c}\text { Actividad física semanal en la } \\
\text { actualidad }\end{array}$} \\
\hline & $\begin{array}{c}\text { No } \\
\text { practica } \\
\text { (a) }\end{array}$ & $\begin{array}{c}\text { 1-2años } \\
\text { (b) }\end{array}$ & $\begin{array}{l}3-4 \\
\text { (c) }\end{array}$ & $\begin{array}{l}5-6 \\
\text { (d) }\end{array}$ & $\begin{array}{c}+6 \\
\text { años } \\
\text { (e) }\end{array}$ & & $\begin{array}{l}1 \text { día } \\
\text { (a) }\end{array}$ & $\begin{array}{l}2-3 \\
\text { (b) }\end{array}$ & $\begin{array}{l}\text { 4-5 } \\
\text { (c) }\end{array}$ & $\begin{array}{c}+5 \text { días } \\
\text { (d) }\end{array}$ & \\
\hline & $\mathrm{M} \pm$ D.t. & $\begin{aligned} & \mathrm{M} \\
& \pm \text { D.t. }\end{aligned}$ & $\begin{aligned} & M \\
& \pm \text { D.t. }\end{aligned}$ & $\begin{aligned} & M \\
& \pm \text { D.t. }\end{aligned}$ & $\begin{array}{c}\text { M } \\
\pm \text { D.t. } \\
\end{array}$ & $p$ & $\begin{aligned} & M \\
& \pm \text { D.t. } \\
&\end{aligned}$ & $\begin{aligned} & M \\
& \pm \text { D.t. }\end{aligned}$ & $\begin{aligned} & M \\
& \pm \text { D.t. }\end{aligned}$ & $M \pm$ D.t. & $p$ \\
\hline & 2.47 & 2.89 & 3.11 & 3.25 & 3.39 & * & 2.91 & 3.12 & 3.63 & 4.15 & ** \\
\hline $\begin{array}{l}\text { Diseñar un } \\
\text { calentamiento }\end{array}$ & \pm 1.01 & \pm .60 & \pm 1.45 & \pm .90 & $\begin{array}{c} \pm .97 \\
a<e(\dagger)\end{array}$ & & \pm .99 & \pm .902 & $\begin{array}{c} \pm .98 \\
a<c(\ddagger) \\
b<c(\ddagger)\end{array}$ & $\begin{array}{c} \pm .80 \\
a<d(\ddagger) \\
b<d(\ddagger)\end{array}$ & \\
\hline $\begin{array}{l}\text { Diseñar mi } \\
\text { programa de } \\
\text { Acond. Físico }\end{array}$ & $\begin{array}{r}1.94 \\
\pm 1.03\end{array}$ & $\begin{array}{r}2.22 \\
\pm .67\end{array}$ & $\begin{array}{r}3.00 \\
\pm 1.41\end{array}$ & $\begin{array}{c}2.5 \\
4 \pm .932\end{array}$ & $\begin{array}{c}2.91 \\
\pm .99 \\
a<e(\dagger)\end{array}$ & ** & $\begin{array}{r}2.46 \\
\pm 1.00\end{array}$ & $\begin{array}{r}2.64 \\
\pm .914\end{array}$ & $\begin{array}{c}3.07 \\
\pm 1.04 \\
a<c(\ddagger) \\
b<c(\ddagger)\end{array}$ & $\begin{array}{c}3.69 \\
\pm 1.03 \\
a<d(\ddagger) \\
b<d(\ddagger)\end{array}$ & ** \\
\hline $\begin{array}{l}\text { Planificar Act. } \\
\text { varios días en } \\
\text { naturaleza }\end{array}$ & $\begin{array}{r}2.29 \\
\pm 1.21\end{array}$ & $\begin{array}{r}2.00 \\
\pm 1.22\end{array}$ & $\begin{array}{r}2.67 \\
\pm \quad .50\end{array}$ & $\begin{array}{r}2.83 \\
\pm 1.24\end{array}$ & $\begin{array}{r}2.79 \\
\pm 1.10\end{array}$ & - & $\begin{array}{r}2.56 \\
\pm 1.24\end{array}$ & $\begin{array}{c}2.53 \\
\pm 1.07 \\
b<c(\ddagger)\end{array}$ & $\begin{array}{r}3.00 \\
\pm .98\end{array}$ & $\begin{array}{r}3.31 \\
\pm 1.25\end{array}$ & * \\
\hline $\begin{array}{l}\text { Planificar } \\
\text { Act. física } \\
\text { naturaleza con } \\
\text { seguridad }\end{array}$ & $\begin{array}{r}2.24 \\
\pm .831\end{array}$ & $\begin{array}{c}2.56 \\
\pm 1.130\end{array}$ & $\begin{array}{r}2.78 \\
\pm .83\end{array}$ & $\begin{array}{r}2.71 \\
\pm 1.08\end{array}$ & $\begin{array}{c}2.81 \\
\pm 1.13\end{array}$ & - & $\begin{array}{r}2.49 \\
\pm 1.20\end{array}$ & $\begin{array}{c}2.56 \\
\pm 1.03 \\
b<c(\ddagger)\end{array}$ & $\begin{array}{c}3.20 \\
\pm 1.02 \\
a<c(\ddagger)\end{array}$ & $\begin{array}{r}2.69 \\
\pm .95\end{array}$ & ** \\
\hline $\begin{array}{l}\text { Participar en } \\
\text { Act. Físicas en } \\
\text { naturaleza }\end{array}$ & $\begin{array}{r}3.00 \\
\pm 1.32\end{array}$ & $\begin{array}{r}3.44 \\
\pm 1.42\end{array}$ & $\begin{array}{r}3.89 \\
\pm 1.05\end{array}$ & $\begin{array}{r}3.38 \\
\pm 1.31\end{array}$ & $\begin{array}{r}3.45 \\
\pm 1.16\end{array}$ & - & $\begin{array}{r}3.21 \\
\pm 1.22\end{array}$ & $\begin{array}{c}3.24 \\
\pm 1.18 \\
b<c(\ddagger)\end{array}$ & $\begin{array}{c}3.80 \\
\pm 1.14 \\
a<c(\ddagger)\end{array}$ & $\begin{array}{r}3.62 \\
\pm 1.12\end{array}$ & $* *$ \\
\hline $\begin{array}{l}\text { Participar en } \\
\text { deportes de } \\
\text { equipo }\end{array}$ & $\begin{array}{r}3.47 \\
\pm 1.23\end{array}$ & $\begin{array}{r}4.00 \\
\pm 1.50\end{array}$ & $\begin{array}{r}4.33 \\
\pm .86\end{array}$ & $\begin{array}{r}4.21 \\
\pm 1.06\end{array}$ & $\begin{array}{r}4.18 \\
\pm .95\end{array}$ & - & $\begin{array}{r}3.60 \\
\pm 1.22\end{array}$ & $\begin{array}{c}4.09 \\
\pm .91 \\
b<c(\ddagger)\end{array}$ & $\begin{array}{c}4.49 \\
\pm .81 \\
a<c(\ddagger)\end{array}$ & $\begin{array}{c}4.85 \\
\pm .38 \\
a<d(\ddagger) \\
b<d(\ddagger)\end{array}$ & $\star *$ \\
\hline $\begin{array}{l}\text { Participar en } \\
\text { algún deporte } \\
\text { individual }\end{array}$ & $\begin{array}{r}2.65 \\
\pm 1.22\end{array}$ & $\begin{array}{r}3.56 \\
\pm 1.24\end{array}$ & $\begin{array}{r}4.11 \\
\pm 1.05\end{array}$ & $\begin{array}{c}3.92 \\
\pm 1.18 \\
\mathrm{a}<\mathrm{d}(\mathrm{t})\end{array}$ & $\begin{array}{c}4.00 \\
\pm 1.08 \\
a<e(\dagger) \\
\end{array}$ & ** & $\begin{array}{r}3.37 \\
\pm 1.30\end{array}$ & $\begin{array}{c}3.82 \\
\pm 1.13 \\
b<c(\ddagger)\end{array}$ & $\begin{array}{c}4.27 \\
\pm .95 \\
a<c(\ddagger)\end{array}$ & $\begin{array}{c}4.46 \\
\pm .66 \\
a<d(\ddagger) \\
\end{array}$ & $\star \star *$ \\
\hline $\begin{array}{l}\text { Organizar una } \\
\text { competición } \\
\text { deportiva o } \\
\text { juegos }\end{array}$ & $\begin{array}{r}2.47 \\
\pm 1.01\end{array}$ & $\begin{aligned} & 3.4 \\
\pm & 1.42\end{aligned}$ & $\begin{array}{c}3.33 \\
\pm 1.12\end{array}$ & $\begin{array}{r}2.92 \\
\pm 1.25\end{array}$ & $\begin{array}{c}3.23 \\
\pm 1.04 \\
a<e(\dagger)\end{array}$ & * & $\begin{array}{r}2.98 \\
\pm 1.11\end{array}$ & $\begin{array}{c}2.91 \\
\pm 1.06 \\
b<c(\ddagger)\end{array}$ & $\begin{array}{c}3.50 \\
\pm 1.00 \\
a<c(\ddagger)\end{array}$ & $\begin{array}{c}3.85 \\
\pm 1.07 \\
b<d(\ddagger)\end{array}$ & ** \\
\hline $\begin{array}{l}\text { Participar } \\
\text { en deporte } \\
\text { recreativo } \\
\text { al menos } \\
\text { una vez a la } \\
\text { semana }\end{array}$ & $\begin{array}{r}3.18 \\
\pm 1.42\end{array}$ & $\begin{array}{r}2.89 \\
\pm 1.36\end{array}$ & $\begin{array}{c}4.00 \pm \\
1.00\end{array}$ & $\begin{array}{c}3.63 \pm \\
1.24\end{array}$ & $\begin{array}{l}3.96 \pm \\
1.058\end{array}$ & * & $\begin{array}{c}3.00 \pm \\
1.27\end{array}$ & $\begin{array}{c}3.84 \pm \\
1.07 \\
a<b(\ddagger) \\
b<c(\ddagger)\end{array}$ & $\begin{array}{c}4.36 \pm \\
.72 \\
a<c(\ddagger)\end{array}$ & $\begin{array}{c}4.54 \pm \\
.776 \\
a<d(\ddagger)\end{array}$ & * \\
\hline $\begin{array}{l}\text { Organizar y } \\
\text { participar en } \\
\text { actividades } \\
\text { con } \\
\text { coreografías } \\
\text { de danza y } \\
\text { baile }\end{array}$ & $\begin{array}{r}2.94 \\
\pm 1.34\end{array}$ & $\begin{array}{r}2.89 \\
\pm .928\end{array}$ & $\begin{array}{r}3.44 \\
\pm 1.01\end{array}$ & $\begin{array}{r}3.42 \\
\pm 1.14\end{array}$ & $\begin{array}{r}3.39 \\
\pm 1.27\end{array}$ & - & $\begin{array}{r}3.37 \\
\pm 1.25\end{array}$ & $\begin{array}{r}3.39 \\
\pm 1.25\end{array}$ & $\begin{array}{r}3.36 \\
\pm 1.30\end{array}$ & $\begin{array}{r}2.77 \\
\pm .72\end{array}$ & - \\
\hline
\end{tabular}




\section{DISCUSIÓN}

Los estudios de maestro de Educación Primaria con especialización en educación física son solicitados principalmente por los hombres, mientras que las mujeres prefieren otras especialidades; resultados similares se han encontrado en otros estudios (PORTO, 2009; ROMERO; ZAGALAZ; ROMERO; MARTÍNEZ, 2011; ZURITA; DELGADO, 2003), que concuerdan además con los estereotipos por sexo en la práctica de actividad física observados en la población española (RODRÍGUEZ-HERNÁNDEZ; FEU; MARTÍNEZ-SANTOS; DE LA CRUZ-SÁNCHEZ, 2011; VARO et al., 2003). En la Educación Secundaria también se ha observado una presencia mayoritaria de docentes hombres en el área de educación física (CAMPOS; GONZÁLEZ; JIMÉNEZ-BEATTY, 2012). Para Porto (2009) las diferencias entre sexo en la elección de especialidades de maestro es un problema estructural y no sólo coyuntural pues esta tendencia se observa longitudinalmente.

Los estudiantes que han elegido la especialidad de maestro de educación física realizan más actividad física semanal que los que quieren realizar otras especialidades. En la población española de 4 a 18 años de edad, los hombres realizan más actividad física semanal que las mujeres (RODRÍGUEZ-HERNÁNDEZ et al. 2011; VARO et al., 2003), por tanto es posible que esto favorezca una mayor proporción de hombres que de mujeres que quieran ingresar en esta profesión. Por otro lado, es un factor a tener en cuenta ya que el hecho de estar vinculado a actividades físico deportivas puede condicionar la interpretación del currículo de educación física (CURTNER-SMITH, 1999).

Por otro lado, los estudiantes que más práctica de actividad física desarrollaron en la infancia y adolescencia parecen estar más motivados para realizar la especialidad de maestro de educación física aunque en nuestro estudio no se han encontrado diferencias significativas. En la literatura científica se ha podido comprobar que los potenciales profesores de educación física manifiestan haber participado en el deporte escolar cuando eran jóvenes (CURTNERSMITH, 2001; HUTCHINSON, 1993; PLACEK et al., 1995) y que esto influye en sus creencias sobre la profesión (CURTNER-SMITH, 1999). Algunos estudios han concluido que entre los motivos de acceso a los estudios de magisterio de educación física figura el gusto por la práctica deportiva y por la imposibilidad de hacer estudios específicos de ciencias del deporte (CAMINA; SALVADOR, 2007).

\subsection{Capacidades adquiridas en la etapa escolar para la práctica de actividad física}

Los estudiantes, en general, manifestaron tener más capacidades para los deportes de equipo, los deportes individuales y para participar al menos una vez en semana en el deporte recreativo; por el contrario indican tener menos capacidades para diseñar actividades físicas en el medio natural y diseñar un programa de acondicionamiento físico.

El género es determinante en la auto percepción para desarrollar actividades físicas y deporte, los chicos se sienten más competentes que las chicas en la mayoría de ítems, excepto en la organización de actividades coreográficas de danza o baile, donde las chicas se sienten más capaces; este resultado confirma una visión estereotipada de la idoneidad de ciertas actividades en función del género, visión que la literatura científica ha verificado que está instalada tanto en el alumnado (ALVARIÑAS; FERNÁNDEZ; LÓPEZ, 2009; BLÁNDEZ; 
FERNÁNDEZ-GARCÍA; SIERRA, 2007; MEANEY; DORNIER; OWENS, 2002) como en el profesorado en servicio (SÁEZ-LÓPEZ; SICILIA; MANZANO, 2010). Los escolares, chicos o chicas, que rompen las barreras para participar de forma plena en cualquier actividad suelen encontrar la desaprobación en las personas de su entorno (BLÁNDEZ et al., 2007).

Las actividades expresivas no sólo gozan de una baja presencia en las planificaciones docentes de educación primaria y secundaria (SICILIA; SÁENZ-LÓPEZ; MANZANO; DELGADO, 2009), a pesar de estar presentes en un bloque de contenidos del diseño curricular base, sino que los libros de texto de educación física para los escolares españoles prestan un mayor interés a los contenidos relacionados con la condición física, los juegos y deportes, superando siempre a la expresión corporal (GONZÁLEZ, 2005). Todo parece jugar en contra de favorecer cambios para una educación menos estereotipada desde una perspectiva de género.

Dado que nuestros resultados corroboran que los hombres se ven con más capacidades para la práctica de los deportes y las actividades relacionadas con condición física y que las chicas se autoperciben con más capacidades para las actividades expresivas es posible que los estereotipos de género puedan ser reproducidos por los docentes. Por tanto, en la formación inicial de los futuros docentes será necesario trabajar sobre las ideas preconcebidas del alumnado para eliminar la idea de una práctica estereotipada por sexo, tanto en la educación física como en el deporte extraescolar.

La cantidad de práctica físico deportiva extracurricular realizada en la edad escolar no influye en la mayoría de las capacidades adquiridas para la práctica autónoma de actividad física, excepto para la práctica de un programa de acondicionamiento físico, la elaboración del calentamiento y la práctica de deportes individuales. Los años de práctica de actividad físico deportiva no condicionan la elección de la especialidad, pero las capacidades que manifiestan haber adquirido en la edad escolar si influyen en la elección de la especialidad de educación física. Este aspecto debe hacernos reflexionar en que lo importante es qué tipo de práctica y cómo y dónde se realiza y con qué finalidad..

La actividad físico - deportiva semanal que realizan los estudiantes del Grado de Maestro en el momento de la elección de la especialidad se asocia con la adquisición en la mayoría de capacidades para la práctica de actividad física, salvo en las capacidades expresivas como el baile, danza,..., y estas influyen en la elección de la especialidad de educación física. Esto confirma que uno de los motivos para elegir la especialidad de educación física es el gusto por la práctica deportiva de los futuros docentes (MEDINA; FERNÁNDEZ; SÁENZ-BUÑUEL, 2013). En este estudio los estudiantes que realizan más práctica de actividad física se sienten más capaces en la mayoría de criterios presentados y son los que optan por la especialidad de educación física. En estudios sobre las motivaciones de los estudiantes de magisterio para elegir la especialidad de educación física está la creencia por parte de los alumnos de la relación de estos estudios con el deporte (CONTRERAS; RUIZ; ZAGALAZ; ROMERO, 2002; GIL, 1998; MEDINA; DELGADO, 1998), de hecho algunos estudiantes justifican el acceso a estos estudios por la imposibilidad de acceder a los estudios de ciencias del deporte (CAMINA; SALVADOR, 2007).

Los resultados muestran claramente que el alumnado que tiene mayores capacidades en todos los ítems, excepto en las habilidades expresivas, se ha decidido por la especialidad de educación física, mientras que los que tienen más capacidades expresivas se han decantado por las otras especialidades. En esta línea, estudios como el de Camina y Salvador (2007) 
constatan que entre el 70 y $80 \%$ de los estudiantes de magisterio de educación física se auto valoraban muy por encima de la media en resistencia a la fatiga y en agilidad para el deporte que los estudiantes de otras especialidades. Además la creencia de tener de altas capacidades para la práctica deportiva podría ser un leitmotiv para sentirse competente para enseñar en la educación primaria (VALTONEN et al., 2015), sobre todo por desconocimiento de la diversidad y proporción de contenidos que el currículum de educación física propone enseñar a lo largo de esta etapa.

Teniendo en cuenta que los estudiantes que han seleccionado la especialidad de educación física puntúan más alto en casi todas las capacidades analizadas, podemos inferir que su idea de educación física podría verse influenciada por los aprendizajes recibidos en su formación previa. En estos casos, el problema podría venir porque muchos aprendizajes deportivos extraescolares se realizan con una clara desconexión de los fines educativos de la educación física (GONZÁLEZ; CAMPOS, 2010).

En los programas formativos del Grado de Maestro con especialización en educación física es necesario considerar y reflexionar sobre las ideas implícitas que las prácticas de actividad física y deportiva previas generan en los estudiantes, y de cómo las vivencias del pasado aportan motivación a su desarrollo como profesional de la educación física. La motivación y el pensamiento reflexivo es imprescindible para aprender de la experiencia y valorarla (FURLOG, 2002), mientras que la ausencia de la misma y la privación del proceso de reflexión sobre lo vivenciado puede distorsionar la idea de una educación física integral y para todos.

La socialización adquirida través de las experiencias vividas en el ámbito extraescolar es particularmente fuerte en los docentes de educación física (CAPEL, 2007). Curtner-Smith (1999) encontró que en la interpretación que los profesores de educación física hacen del currículum del área está influenciada, entre otros factores, por el género, la actividad física y el deporte y la participación en la educación física y el deporte escolar durante la infancia y la adolescencia. Por ello, es necesario trabajar con el alumnado sobre sus ideas implícitas sobre la educación física, de forma que podamos evitar la reproducción de un modelo de enseñanza preconcebido que pueda prevalecer si el proceso de enseñanza aprendizaje de los futuros docentes se desarrolla a través de un modelo técnico e irreflexivo (CAPEL, 2007; CRUM, 1994). Nuestros resultados vienen a recordar la necesidad de indagar en las creencias y conocimientos previos que tienen los futuros docentes (GROSSMAN; WILSON; SHULMAN, 2005; WOOLFOLK; MURPHY, 2001), y desarrollar la formación de estos acompañada de procesos de reflexión sobre la propia práctica como docentes y sus ideas implícitas sobre la profesión docente (SCHÖN, 1983).

Como prospectiva de esta investigación se sugiere la necesidad de estudiar, desde una perspectiva cualitativa y cuatitativa, como influye en la elección de la especialidad de maestro de educación física variables como: los tipos de prácticas físico deportivas realizadas tanto dentro como fuera de la escuela, la satisfacción con estas prácticas, la finalidad del proceso formativo, la posible influencia de los docentes, técnicos deportivos, familia y los iguales,... Además se debería analizar cómo influyen estas variables en la formación de las competencias para una práctica física autónoma en el momento de la elección de la especialidad de maestro de educación física, pues se ha demostrado que la autopercepción de ser competente para la práctica físico deportiva es un motivo para elegir la especialidad de educación física. 


\section{REFERENCIAS}

ALVARIÑAS, Myriam; FERNÁNDEZ, María Ángeles; LÓPEZ, Cristina. Actividad física y percepciones sobre deporte y género. Revista de Investigación en Educación, Vigo, v. 6, p. 113-122, 2009.

ALVIRA, Francisco. La encuesta: una perspectiva general metodológica. Madrid: Centro de Investigaciones Sociológicas, 2004.

ATO, Manuel; LÓPEZ, Juan J.; BENAVENTE, Ana. Un sistema de clasificación de los diseños de investigación en psicología. Anales de psicología, Murcia, v. 29, n.3, 1038-1059, 2013.

BLÁNDEZ, Julia; FERNÁNDEZ-GARCÍA, Emilia; SIERRA, Miguel Ángel Estereotipos de género, actividad física y escuela: la perspectiva del alumnado. Revista de currículum y formación del profesorado, Granada, v. 2, p. 1-21, 2007.

BULGER, Sean M.; HOUSNER, Lynn D. Modified delphi investigation of exercise science in physical education teacher education. Journal of Teaching in Physical Education, n. 26, p. 57-80, 2007.

BURKE, Peter J. Teacher Development: Induction, Renewal and Redirection. Londres: The Falmer, 1987.

CAMINA, Asunción; SALVADOR, María Isabel. Condicionantes y Características de los Estudiantes que Inician Magisterio. Estudio Descriptivo y Comparativo entre Especialidades. Tendencias Pedagógicas, Madrid, n. 12, p. 245-262, 2007.

CAMPOS, Antonio; GONZÁLEZ, María Dolores; JIMÉNEZ-BEATTY, José Emilio. El perfil profesional del profesorado de educación física en educación secundaria en la comunidad valenciana.

EDUCACIÓN XXI, Madrid, v. 15, n. 1, p. 135-155, 2012. http://dx.doi.org/10.5944/educxx1.15.1.153

CAPEL, Susan. Moving beyond physical education subject knowledge to develop knowledgeable teachers of the subject. The Curriculum Journal, v. 18, n. 4, p. 493-507, 2007.

COLOM, Antonio J. Continuidad y complementariedad entre la formación formal y no formal. Revista de Educación, Madrid, n. 338, p. 9-22, 2005.

CONTRERAS, Onofre R.; RUIZ, Luis Miguel; ZAGALAZ, María Luisa; ROMERO, Santiago. Las creencias en la formación inicial del profesorado de Educación Física. Incidencias en la transformación de su Pensamiento. Revista Interuniversitaria de Formación de Profesorado, Zaragoza, n. 45, p. 131-149, 2002.

CRUM, Bart. Physical Education Teacher. Weak points chances for improvement. Paper presented at the Fifth EUPEA-Forum. Coimbra: EUPEA, 1994.

CURTNER-SMITH, Mattew. D. The more things change the more they stay the same: factors influencing teachers' interpretations and delivery of National Curriculum Physical Education. Sport, Education and Society, v. 4, n. 1 p. 75-97, 1999.

CURTNER-SMITH, Mattew. D. The Occupational Socialization of a First-Year Physical Education Teacher with a Teaching Orientation. Sport, Education and Society, v. 6, n. 1, p.81-105, 2001.

EIRÍN, Raul; GARCÍ-RUSO, Herminia. M.; MONTERO, Lourdes. Profesores principiantes e iniciación profesional. Estudio exploratorio. Revista de Curriculum y Formación del Profesorado, Granada. v. 13, n. 1, 2009. Disponible en: < https://www.ugr.es/ recfpro/rev131ART7.pdf>. Acceso en: 1oct. 2015.

FEU, Sebastián; IBÁÑNEZ, Sergio José; LORENZO, Alberto; JIMÉNEZ, Sergio; CAÑADAS, María. El conocimiento profesional adquirido por el entrenador de balonmano: experiencias y formación.

Revista de Psicología del Deporte, Palma, v. 21, n. 1, p. 107-115, 2012. 
FEU, Sebastián; IBÁÑEZ, Sergio José; GOZALO, Margarita. La formación inicial de los entrenadores de balonmano para la enseñanza del deporte en la edad escolar. Cultura, Ciencia y Deporte, Murcia, v. 14, n. 6, p. 109-117, 2010.

FURLONG, J. La intuición y la crisis de la profesionalidad entre los docentes. En: ATKINSON, Terry; CLAXTON, Guy (Ed.). El profesor intuitivo. Barcelona: Octaedro, 2002. p. 29-49.

GIL, Pedro. Evaluación contextual. El estudio de un caso. El plan de estudios, currículum, de la formación inicial del Maestro Especialista en Educación Física en la Escuela de Magisterio de Albacete. U.C.L.M. En: GARCÍA, A. ; RUIZ, Francisco; CASIMIRO A. J. La enseñanza de la Educación Física y el Deporte Escolar. Almería: IAD, 1998. p. 167-170.

GONZÁLEZ, María D.; CAMPOS, Antonio. La intervención didáctica del docente del deporte escolar, según su formación inicial. Revista de Psicodidáctica, v. 15, n. 1, p. 101-120, 2010.

GONZÁLEZ, M. ¿Tienen sexo los contenidos de la Educación Física Escolar? Transmisión de estereotipos de sexo a través de los libros de texto en la etapa de Secundaria. Revista Internacional de Medicina y Ciencias de la Actividad Física y el Deporte, v. 5, n. 18, p. 77-88, 2005.

GROSSMAN, Pamela. L.; WILSON, Suzzane M.; SHULMAN, Lee. S. Profesores de sustancia: el conocimiento de la materia para la enseñanza. Revista de currículum y formación del profesorado, v. 9, n. 2, p. 1-24, 2005. Disponible en: < https://www.ugr.es/ recfpro/rev92ART2.pdf >. Acceso en: 1oct. 2015.

HALL, John. Initial Teacher Education: Specialists and Generalists - A review of the Literature. Glasgow: Scottish Council for Research in Education, 2000.

HUTCHINSON, Gayle. Prospective teachers' perspectives on teaching physical education: an interview study on the recruitment phase of teacher socialization. Journal of Teaching in Physical Education, v. 12, n. 4, p. 344-354, 1993.

JORDELL, Karl. Problems of Beginning and more Experienced Teachers in Norway. Scandinavian Journal of Educational Research, v. 29, n. 3, p. 105-121, 1987.

LATORRE, María José. El entrenamiento práctico universitario de los futuros profesionales de la educación musical: aproximación a sus creencias desde la Universidad de Granada. Revista de Psicodidáctica, Leioa, v. 12, n. 2, p. 257-268, 2007.

LOHMAN, Margaret $C$. Factors influencing teachers' engagement in informal learning activities. Journal of Workplace Learning, Bingley, v. 18, n. 3, p. $141-156,2006$.

MEANEY, Karen S.; DORNIER, Lanie; OWENS, Mary S. Sex-Role Stereotyping for Selected Sport and Physical Activities across Age Groups. Perceptual and Motor Skills, Missoula, v. 94, p. 743-749, 2002.

MEDINA, Jesús; DELGADO-NOGUERA, Miguel Ángel. Una primera aproximación a la investigación sobre las teorías del alumno acerca de la Educación Física. En: GARCÍA, A.; RUIZ, F.; CASIMIRO, A. J. (Eds.), La Enseñanza de la Educación Física y el Deporte Escolar. Almería: IAD, 1998. p. 446450.

MONTERO, Ignacio; LEÓN, Orfelio. A guide for naming research studies in Psychology. International Journal of Clinical and Health Psychology, Granada, v. 7, n. 3, p. 847-862. 2007.

NUNNALLY, Jum C.; BERNSTEIN, Ira H. Psychometric Theory. 3.ed.. New York: McGraw Hill, 1994. O'BRYANT, Camille P.; O'SULLIVAN, Mary; RAUDENSKY, Jeanne. Socialization of prospective physical education teachers: The story of new blood. Sport, education and society, v. 5, n. 2, p. 177193, 2000. 
PLACEK, Judith; DODDS, Patt; DOOLITTLE, Sarah; PORTMAN, Penelope; RATLIFFE, Thomas; PINKMAN, Kathy. Teaching recruits' physical education backgrounds and beliefs about purposes for their subject matter. Journal of Teaching in Physical Education, v. 14, n. 3, p. 246-261, 1995.

PORLÁN, Rafael; RIVERO, Ana. El conocimiento de los profesores. Sevilla: Diada, 1998.

PORTO, Benxamín. Feminización y masculinización en los estudios de maestro y educación física en Galicia. Revista de Investigación en Educación, Vigo, n. 6, p. 50-57, 2009.

RANDALL, Lynn; MAEDA, Julienne K. Pre-Service Elementary Generalist Teachers' Past Experiences in Elementary Physical Education and Influence of These Experiences on Current Beliefs. Brock Education: a Journal of Educational Research and Practice, Hamilton, v.19, n. 2, p. 20-35, 2010.

RODRÍGUEZ, María del Carmen; FERNÁNDEZ, Carmen María. Educación formal, no formal e informal en el Espacio Europeo: nuevas exigencias para los procesos de formación en educación. Aula abierta, Oviedo, n. 85, p. 45-56, 2005.

RODRÍGUEZ-HERNÁNDEZ, Arturo; FEU, Sebastián; MARTíNEZ-SANTOS, Raúl; DE LA CRUZSÁNCHEZ, Ernesto. Prevalence and distribution of inactivity and weight excess in Spanish scholar children. E-balonmano.com: Revista de Ciencias del Deporte, Mérida, v. 7, n. 3, p. 157-168, 2011.

ROMERO Cipirano; ZAGALAZ, María Luisa; ROMERO, Miriam Noemí; MARTíNEZ, Emilio. J. Importancia de las competencias profesionales de los Maestros en Educación Física expresadas por los estudiantes. Retos. Nuevas tendencias en educación física, deporte y recreación, San Javier, n. 19, p. 62-68, 2011.

RYAN, Stu; BRIDGES, Stephen; YERG, Beverly. The influence of teacher education on teachers' beliefs about purposes of physical education. Education, v. 121, n. 2, p. 301-304, 2000.

SÁENZ-LÓPEZ BUÑUEL, Pedro; SICILIA, Álvaro; MANZANO, J.I.. La opinión del profesorado sobre la enseñanza de la educación física en función del género. Revista Internacional de Medicina y Ciencias de la Actividad Física y el Deporte, Madrid. v. 10, n. 37, p. 167-180, 2010. Disponible en: < http://cdeporte.rediris.es/revista/revista37/artopinion148.htm>. Acceso en: 5 oct. 2015.

SCHÖN, Donald. The reflective practitioner: How professionals think in action. New York: Basic Books, 1983.

SHKEDI, Asher; LARON, Dina. Between idealism and pragmatism: a case study of student teachers' pedagogical development. Teaching and Teacher Education, v. 20, n. 7. p. 693-711, 2004.

SICILIA, Álvaro; SÁENZ-LÓPEZ, Pedro; MANZANO José Ignacio; DELGADO, Miguel Ángel. El desarrollo curricular de la Educación Física en Primaria y Secundaria: un análisis desde la perspectiva del profesorado. Apunts, Educación Física y Deportes, n. 98, p. 23-32, 2009. Disponible en: < http:// www.revista-apunts.com/es/hemeroteca?article=1390 >. Acceso en: 5 oct. 2015.

VALTONEN, Juha; REUNAMO, Jyrki; HIRVENSALO, Mirja; RUISMÄKI, Heikki. Socialization Into Teaching Physical Education - Acculturative Formation Of Perceived Strengths. The European Journal of Social and Behavioural Sciences, v. 12, p. 1683-1695, 2015.

VAN MAANEN, John; SCHEIN, Edgar. Toward a theory of organizational socialization. In: STAW, Barry (Ed.). Research in organizational behavior, Greenwich, CT: JAI, 1979. p. 209-261.

VARO, José J.; MARTíNEZ-GONZÁLEZ, Miguel A.; DE IRALA-ESTÉVEZ, Jokin; KEARNEY, John; GIBNEY, Michael; MARTíNEZ, J. Alfredo. Distribution and determinants of sedentary lifestyles in the European Union. International Journal of Epidemiology, v. 32, n. 1, p. 138-146, 2003.

WOOLFOLK, Anita; MURPHY, P. Karen. (2001). Teaching Educational Psychology to the Implicit Mind. In: TORFF, Bruce; STERNBERG, Robert. Understanding and Teaching the Intuitive Mind:

Student and Teacher Learning. London: Lawrence Erlbaum Associates, 2001. p. 145-185. 
ZURITA, Félix; DELGADO, Miguel Ángel. Estudio de la teorías implícitas de la educación física e la formación inicial de los maestros en las diferentes especialidades, ¿Qué opinan los futuros maestros? Kronos: Revista universitaria de la actividad física y el deporte,Villaviciosa de Odón, n. 5, p. 2738, 2003. 